\title{
The TNM classification of breast cancer: need for change
}

\author{
Paolo Arnone $\cdot$ Stefano Zurrida $\cdot$ Giuseppe Viale $\cdot$ \\ Silvia Dellapasqua $\cdot$ Emilia Montagna $\cdot$ \\ Paola Arnaboldi · Mattia Intra · Umberto Veronesi
}

Published online: 6 July 2010

(C) Springer-Verlag 2010

\section{Background}

The need for a unified and internationally accepted cancer staging system was recognized in the first half of the twentieth century and led to the publication of the first International Union Against Cancer (UICC) [1] staging system in the 1950s. This was followed by the International Federation of Gynecology and Obstetrics (FIGO) classification of women's cancers [2] and the American Joint

This article is an extended and modified version of the one published previously in J Clin Oncol 2009; 27(15):2427-2428.

Permission has been granted by Prof. Umberto Veronesi, Scientific Director of European Institute of Oncology to reuse the information again for this article.

P. Arnone $(\bowtie) \cdot S$. Zurrida $\cdot$ M. Intra

Senology Division, European Institute of Oncology,

Via Giuseppe Ripamonti 435, 20141 Milan, Italy

e-mail: paolo.arnone@ieo.it

S. Zurrida - U. Veronesi

European Institute of Oncology, Milan, Italy

\section{S. Zurrida $\cdot$ G. Viale}

Faculty of Medicine and Surgery,

University of Milan, Milan, Italy

G. Viale

Department of Pathology and Laboratory Medicine,

European Institute of Oncology, Milan, Italy

S. Dellapasqua $\cdot$ E. Montagna

Department of Medical Oncology,

European Institute of Oncology, Milan, Italy

P. Arnaboldi

Unit of Psychological Oncology,

European Institute of Oncology, Milan, Italy
Committee for Cancer (AJCC) classification, the first edition of which was published in 1977 [3].

These manuals are based on clinical and pathological data and make it possible to assign a stage to a malignancy that indicates the extent of the disease, and also provides indications for treatment and prognosis. The UICC TNM classification $\left(\mathrm{TNM}_{\mathrm{UICC}}\right)$ considers the size of the primary (T), regional lymph node status $(\mathrm{N})$, and presence of distant metastases (M) as the fundamental disease characteristics.

Although the TNM classification has been regularly updated since its first edition in 1977 (new 7th edition published in December 2009), the $\mathrm{TNM}_{\mathrm{UICC}}$ classification of breast cancer is, in our opinion, obsolete and requires radical overhaul, with the introduction of new information to produce a more modern and useful characterization of breast tumours.

Based on the experience of an interdisciplinary work group, which over the last 10 years examined over 30,000 breast cancer patients, the European Institute of Oncology has produced proposals for a revision of the TNM classification of breast cancers.

One of motives that inspired the new classification was the language of the existing $\mathrm{TNM}_{\mathrm{UICC}}$ which often has a negative psychological impact on the patient receiving the diagnosis. Words like "malignancy", "carcinoma", and "infiltrating" are particularly at fault here. Psychological problems are present in around $20-40 \%$ of persons diagnosed with cancer [4] and the emotive terminology used by the physician or in the diagnostic report-which conjures up images of pain, suffering, and death-are likely to exacerbate these problems.

The fear and uncertainty that a cancer diagnosis generates may motivate the person to put her faith in the treating physician, ask questions about the illness, and find out more on the Internet. But the opposite reaction of 
withdrawing into oneself and experiencing the diagnosis is a death sentence, is common [5]. The way the diagnosis is communicated, by the physician or the diagnostic report, can markedly influence how the patient reacts. The terminology used by the TNM UICC $_{\text {classification can exacerbate }}$ these problems and make it more difficult for the patient to come to terms with her disease [6].

When a patient is told she has "carcinoma in situ" she will usually become extremely anxious, thinking she has an incurable cancer. Consider a woman diagnosed with "infiltrating carcinoma". The already traumatic effect of the word "carcinoma" is worsened by its qualifier "infiltrating". The woman understands that, even though her illness may have been diagnosed at an early stage, it has already "infiltrated" her organs and will surely kill her. The result is that the patient has none of the hope necessary to face her treatment program, asks how long she has to live, and is beside herself with the thought she will not see her children grow up.

After an interview with the physician the patient with in situ carcinoma is reassured, learning she has ductal or lobular carcinoma in situ; that this type of lesion differs biologically from infiltrating carcinoma; and that she is likely to be completely cured. The second woman will also benefit from more information about the nature of her disease.

One of the important innovations of the European Institute of Oncology TNM classification $\left(\mathrm{TNM}_{\mathrm{IEO}}\right)$ is to eliminate the terms "in situ carcinoma" and "infiltrating" - which are likely to be misconstrued by the patientand replace them with a more logical and accurate terminology [7-9]. The new terminology also clearly indicates the difference between lesions that can metastasize and those which do not.

The second major innovation proposed by the $\mathrm{TNM}_{\mathrm{IEO}}$ classification is to specify $\mathrm{T}$ and $\mathrm{N}$ more precisely, with the advantages of furnishing a more exact and useful indication of "disease stage", and at the same time retaining compatibility with the older $\mathrm{T}$ and $\mathrm{N}$ categories and hence permitting statistical comparisons between older data and new data.

An important aim of previous $\mathrm{TNM}_{\mathrm{UICC}}$ classifications was to provide some indication of prognosis and hence also a rough guide to treatment. Ductal carcinoma in situ (DCIS) and lobular carcinoma (LCIS) are included within the $\mathrm{T}$ classification. However, as noted above, these neoplasms are non-invasive by definition and are incapable of metastasizing to distant sites. They should not, therefore, be considered either malignant or carcinoma, and should be excluded from the TNM classification. We propose adopting the classification of Tavassoli [10] which drops the terms DCIS and LCIS, and instead uses ductal intraepithelial neoplasia (DIN) and lobular intraepithelial neoplasia (LIN) (Table 1), and further divides these entities according to the grade of the neoplasia.

Furthermore, since the word "infiltrating" in "infiltrating carcinoma" is redundant as carcinoma is by definition infiltrating (or invasive), we also propose removing "infiltrating" from the TNM classification.

\section{Tumour size (T)}

In the $\mathrm{TNM}_{\mathrm{UICC}}$ classification, cancers are classified into categories (T1, T2, etc.) according to size, but with no real logic to the categories. This approach was reasonable 60 years ago when the first TNM classification was developed. At that time it was only possible to diagnose a breast cancer large enough to be palpated. Today, however, the lack of logic in the $\mathrm{T}$ categories is evident. First, because they do not make full use of the information provided by modern diagnostic methods which allow the identification of ever smaller tumours. Thus, the T1 category ranges from a few millimetres to $2 \mathrm{~cm}$, with a large difference in prognosis between the extremes. These differences in prognosis are in part acknowledged by the $\mathrm{T} 1$ subcategories (T1a, 1-5 mm; T1b, 6-10 mm; and T1c 11$20 \mathrm{~mm}$ ), but these are essentially arbitrary and needlessly complex.

The prognostic variation within $\mathrm{T} 2$, which includes cancers from 2.1 to $5 \mathrm{~cm}$, is even more marked, and further illustrates the arbitrariness of $\mathrm{T}$ categories which consider diameter rather than volume. Thus, a tumour of diameter $2.1 \mathrm{~cm}$ would have a volume $4.5 \mathrm{ml}$ (assuming it were spherical) while a $5 \mathrm{~cm}$ cancer would have volume of about $60 \mathrm{ml}$. The difference in prognosis between the two masses is marked.

We therefore propose abolishing the $\mathrm{T}$ categories of the $\mathrm{TNM}_{\mathrm{UICC}}$ classification and replacing them by the exact specification of tumour size in centimetres (Table 2). The result is a more intuitively comprehensible and information-rich classification.

\section{Regional lymph nodes (N)}

The classification of locoregional lymph node involvement proposed by the $\mathrm{TNM}_{\text {IEO }}$ follows the logic of the new $\mathrm{T}$ classification: it specifies the exact number of lymph nodes found to be metastatic over the total number removed and examined. For example $\mathrm{pN}_{(5 / 21)}$ states that 5 out of the 21 lymph nodes examined were metastatic. Consider the utility of this: if 3 nodes were positive of the 28 axillary lymph nodes removed and examined the $\mathrm{N}$ stage would be $\mathrm{N} 1 \mathrm{~b}$ by $\mathrm{TNM}_{\mathrm{UICC}}$, but simply $\mathrm{pN}+(3 / 28)$ by $\mathrm{TNM}_{\mathrm{IEO}}$. 
Table 1 TNM breast staging comparison guide: sixth UICC versus Tavassoli classification (Primary Tumour (T))

\begin{tabular}{|l|l|l|l|}
\hline \multicolumn{2}{|c|}{ TNM $_{\text {uicc }}$ 6th version (2002) } & \multicolumn{2}{c|}{ Tavassoli Classification } \\
\hline \multirow{3}{*}{ pTis } & pTis (DCIS) - Ductal Carcinoma In Situ. & & \\
\cline { 2 - 4 } & DCIS grade 1 & & \\
\cline { 2 - 4 } & DCIS grade 2 & & \\
\hline & DCIS grade 3 & & Ductal Intraepithelial Neoplasia grade 1c (DIN1c) \\
\hline & pTis (LCIS) - Lobular Carcinoma In Situ. & & Ductal Intraepithelial Neoplasia grade 3 (DIN3) \\
\hline & LCIS (classic) & & Lobular Intraepithelial Neoplasia grade 2 (LIN2) \\
\hline & LCIS (High grade -pleiomorphic) & & \\
\hline & & & \\
\hline
\end{tabular}

Table 2 TNM breast staging comparison guide: sixth UICC versus TNM $_{\text {IEO }}$ (Primary Tumour (T))

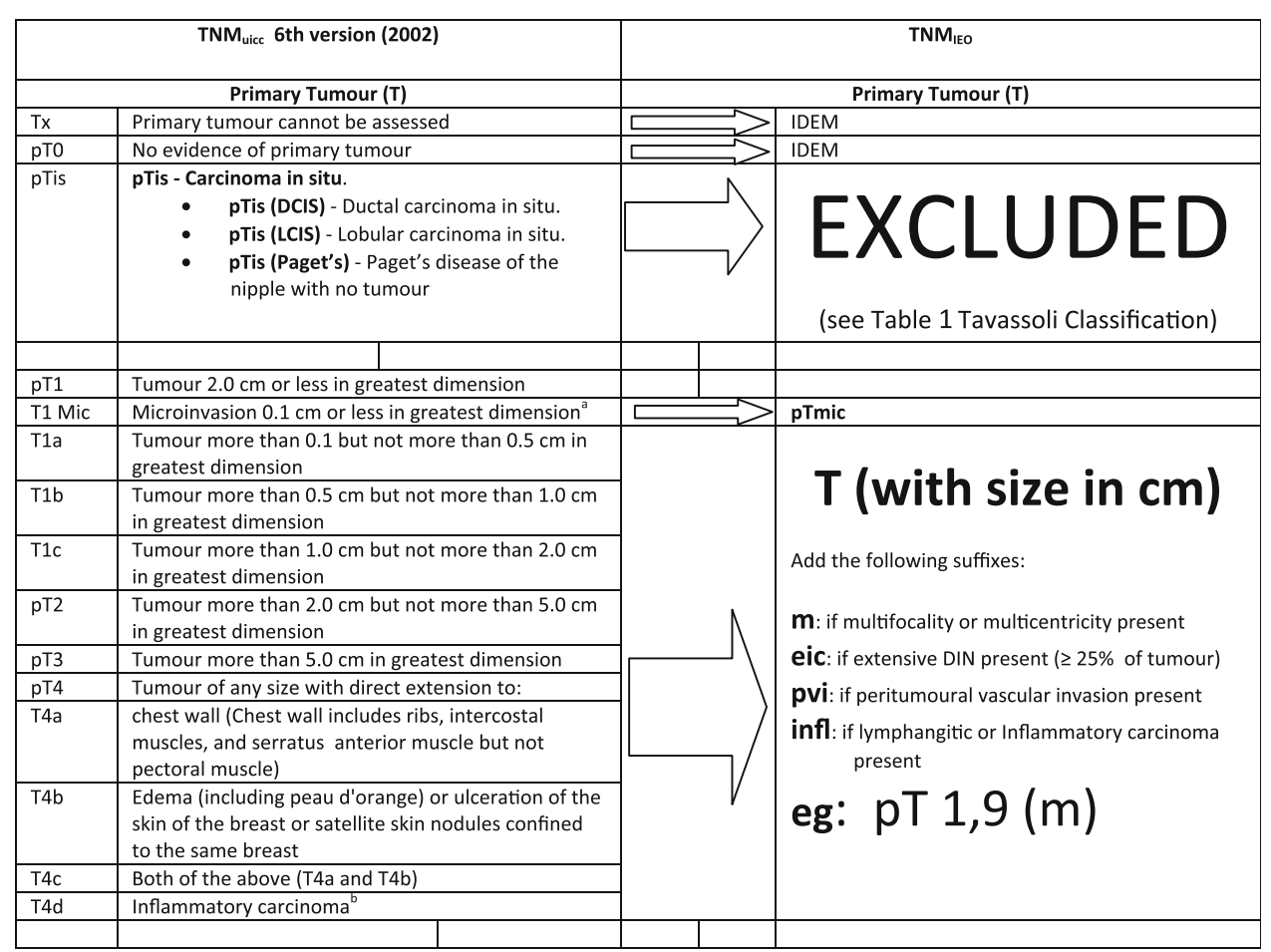

${ }^{\text {a }} \mathrm{T} 1 \mathrm{Mic}$ is defined as microinvasion $0.1 \mathrm{~cm}$ or less in greatest dimension. The presence of multiple tumour foci of microinvasion should be noted in parentheses. Microinvasion is infiltration of neoplastic cell beyond the basal membrane of adjacent tissue without foci greater than $0.1 \mathrm{~cm}$ in max diam. In the presence of multiple micro-invasive foci, classification is based on largest size (not the sum of diameters of several foci). Presence of multiple foci must be noted, as for multiple larger size carcinomas

${ }^{\mathrm{b}}$ Inflammatory carcinoma is a clinicopathologic entity characterized by diffuse brawny induration of the skin of the breast with an erysipeloid edge, usually without an underlying palpable mass. If the skin biopsy is negative and there is no clinically apparent primary tumour, when the clinical diagnosis is inflammatory carcinoma (T4d), the pathological staging should be pTX. Skin depression, nipple retraction or other skin alterations (except those for T4b and T4d) can also be present in T1, T2, and T3 disease and do not change the T category. Radiologically there may be a detectable mass and characteristic thickening of the skin over the breast. This clinical presentation is due to tumour embolization of dermal lymphatics with engorgement of superficial capillaries

Over the last decade sentinel node biopsy has completely revolutionized the surgical approach to the axilla in breast cancer [11]. This revolution has been felt in the $\mathrm{TNM}_{\text {UICC }}$ classification, but the modification proposed by the $\mathrm{TNM}_{\mathrm{IEO}}$ is to simply add the suffix (sn) (Table 3). For a lymph node with extracapsular, invasion the suffix ExCp is added.

Sentinel node biopsy combined with intra-operative examination of $50 \mu \mathrm{m}$ serial sections [12] has led to the frequent finding of micrometastases $(<2 \mathrm{~mm})$ or even of 
Table 3 TNM breast staging comparison guide: sixth UICC versus TNM $_{\text {IEO }}$ (Regional Lymph Nodes (N))

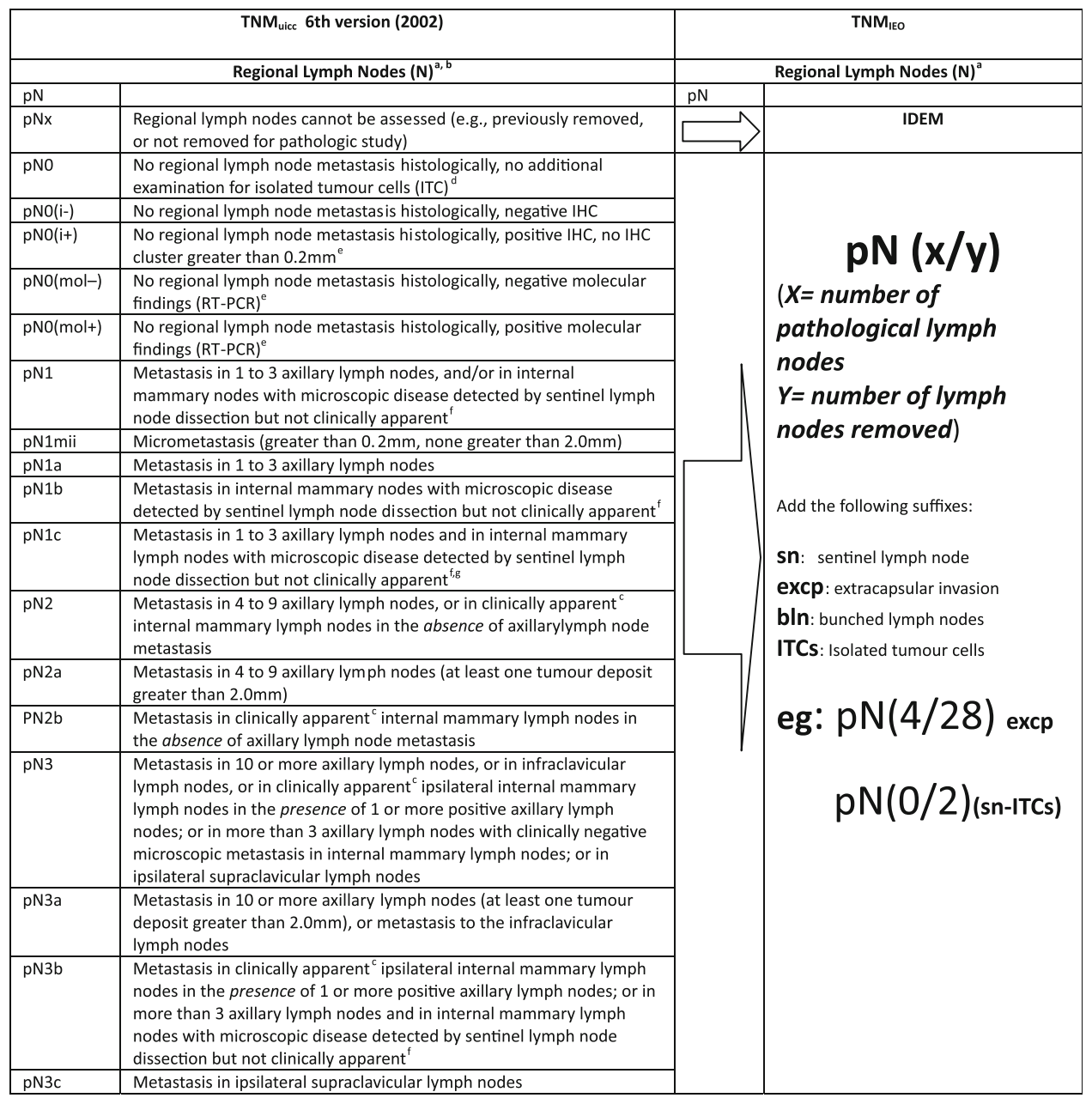

${ }^{a}$ The regional lymph nodes are: (1) Axillary (ipsilateral): interpectoral lymph nodes (Rotter's node) and lymph nodes along the axillary vein and its tributaries, which may be divided into the following three levels: Level 1(lower axilla): lymph nodes lateral to the lateral margin of the pectoralis minor; Level 2 (middle axilla): lymph nodes between the medial and lateral margins pectoralis minor and interpectoral lymph nodes (Rotter's nodes); Level 2 (apex of the axilla): lymph nodes medial to the medial margin of pectoralis minor including those known as subclavicular, infraclavicular or apical Note: intramammary lymph nodes are considered axillary lymph nodes; (2) ipsilateral infraclavicular (subclavicular); (3) mammary internal (ipsilateral): are situated in the intercostal space along the lateral edge of the sternum on the endothoracic fascia; (4) supraclavicular (ipsilateral)

b Classification is based on axillary lymph node dissection with or without sentinel lymph node dissection. Classification based solely on sentinel lymph node dissection without subsequent axillary lymph node dissection is designated (sn) for "sentinel node", e.g., pN0(i+)(sn)

${ }^{c}$ Clinically apparent is defined as detected by imaging studies (excluding lymphoscintigraphy) or by clinical examination or grossly visible pathologically

${ }^{\mathrm{d}}$ Cases with only isolated tumour cells (ITC) in regional lymph nodes are classified pNO. Isolated tumour cells (ITC) are defined as individual tumour cells or small cell clusters not greater than $0.2 \mathrm{~mm}$, usually detected only by immunohistochemical (IHC) or molecular methods but which may be verified on H\&E stains. ITCs do not usually show evidence of metastatic activity (e.g., proliferation or stromal reaction)

e RT-PCR: reverse transcriptase/polymerase chain reaction

${ }^{\mathrm{f}}$ Not clinically apparent is defined as not detected by imaging studies (excluding lymphoscintigraphy) or by clinical examination

$\mathrm{g}$ If associated with greater than 3 positive axillary lymph nodes, the internal mammary nodes are classified as pN3b to reflect increased tumour burden

small clusters of cancer cells (isolated tumour cells). The $\mathrm{TNM}_{\text {UICC }}$ classification was forced to change and the categories $\mathrm{N}_{\text {mic }}$ and $\mathrm{N}_{\text {itc }}$ were introduced and are used for example in the trial 23/01 [13].

\section{Distant metastases (M)}

The $\mathrm{TNM}_{\mathrm{UICC}}$ classification uses M1 to indicate the presence of distant metastasis. We suggest specifying the site 
Table 4 TNM breast staging comparison guide: sixth UICC versus TNM $_{\text {IEO }}$ (Distant Metastasis (M))

\begin{tabular}{|c|c|c|c|c|}
\hline \multicolumn{2}{|r|}{ TNM $_{\text {uicc }}$ 6th version (2002) } & \multicolumn{3}{|r|}{ TNM $_{\text {IEO }}$} \\
\hline \multicolumn{2}{|r|}{ Distant Metastases (M) } & \multicolumn{3}{|c|}{ Distant Metastases (M) } \\
\hline $\mathrm{pM}$ & & & \\
\hline $\mathrm{pMx}$ & Distant metastasis cannot be assessed & \multicolumn{2}{|l|}{$\mathrm{pM}$} & $\begin{array}{l}\text { Distant metastasis cannot be } \\
\text { assessed }\end{array}$ \\
\hline \multirow[t]{6}{*}{ pM0 } & No distant metastasis & \multicolumn{2}{|c|}{$\longrightarrow$} & No distant metastasis \\
\hline & & & pM1 PUL & Lung \\
\hline & & & pM1 oss & Bone \\
\hline & & & pM1 HEP & Liver \\
\hline & & & pM1 BRA & Brain \\
\hline & & & pM1 LYM & Extraregional lymph nodes \\
\hline \multirow[t]{8}{*}{ pM1 } & $\begin{array}{l}\text { Distant metastasis present ( site may be } \\
\text { specified) }\end{array}$ & & PM1 MAR & Bone Marrow \\
\hline & & & pM1 PLE & Pleura \\
\hline & & & pM1 PER & Peritoneum \\
\hline & & & pM1 ADR & Adrenal \\
\hline & & & pM1 SKI & Skin \\
\hline & & & pM1 OTH & Other organs \\
\hline & & & & \\
\hline & & & pM1susp & $\begin{array}{l}\text { Clinical suspicious of metastases } \\
\text { requiring verification }\end{array}$ \\
\hline & & & & \\
\hline
\end{tabular}

of distant disease spread, since different sites can be treated with different possibilities of success using new treatment modalities, such as radiofrequency thermoablation [14], stereotactic radiosurgery (gamma knife) [15], high-intensity focused ultrasound [16], and monoclonal antibodies $[17,18]$. We propose using suffixes to indicate the metastatic sites. Table 4 illustrates the use of $\mathrm{M}$ suffixes proposed by the $\mathrm{TNM}_{\mathrm{IEO}}$ in comparison with the current $\mathrm{TNM}_{\text {UICC }}$ system.

\section{Biological factors}

As molecular and genetic understanding of breast cancer pathology has increased, new biological characteristics have been identified as prognostic indicators, and new adjuvant treatments have been developed. This has resulted in an increasingly personalized approach to breast cancer treatment that takes into account the diverse biological characteristics of the individual patient and her disease.

Examples include the expression by cancer cells of nuclear receptors for estrogens (ER) and for progesterone; expression of the Ki-67 marker of cell proliferation [19] present in active phases of cell cycle (G1, S, G2, and mitosis), but absent from resting cells (GO) [20]; and more recently expression of human epidermal growth factor receptor 2 (HER2).

HER2 (also known as neu or erbB2) is overexpressed in approximately 20-30\% of breast cancers, usually because gene duplication events increase the number of copies of the HER2 gene in each cancer cell. HER2 overexpression indicates an aggressive type of breast cancer, and the relatively new monoclonal antibody drug trastuzumab is able to inactivate HER2 and improve the prognosis for this type of breast cancer.

There is some evidence that the way HER2 expression is measured in breast cancer samples can influence the indication for trastuzumab use [21]. HER2 expression is most often assessed immunohistochemically (IHC), assigning a score of 0 to +3 according to the intensity and completeness of staining on the cancer cell membrane [22, 23]. The second method, typically used if the IHC result is uncertain, employs fluorescent in situ hybridization (FISH) to estimate the number of copies of the HER2 gene per cancer cell. The HER2 gene is present on the long arm of chromosome 17 (17q21-q22) [24] and HER2 gene copy number is usually expressed relative to chromosome 17 . Unfortunately, notwithstanding efforts to introduce a standardized and reliable HER2 assay method, the result may sometimes be uncertain, introducing uncertainties in the indication for trastuzumab use.

We propose introducing HER2 into the $\mathrm{TNM}_{\mathrm{IEO}}$ classification, specifying not only the ICH score (indication of percentage of cancer cells with highly positive staining), but also the result of the FISH analysis, if performed, specifying the amplification/non-amplification/polysomy of chromosome 17, and including the absolute or relative number of copies of the HER2 gene.

Another important consideration regarding the role of cancer stem cells in cancer spread. According to the cancer stem cell theory a small number of cancer stems cells are present in most tumours. These are relatively slow growing and divide asymmetrically to produce more self-renewing stem cells, but mainly the quickly dividing cells that form the mass of the tumour. Although these latter cells are variably differentiated they do not possess metastatic 
potential. According to this view, the cells in a cancer are hierarchically organized, as in normal tissue, and the carcinogenetic process can be regarded as organogenesis gone wrong. The quantity of stems cells and their detailed properties are likely to vary between one cancer and another accounting for differences in expansion kinetics. It has been suggested for example that the "basal-like type" of breast cancer has an evaluated number of cancer stem cells $[25,26]$.

A cancer with a high proportion of cancer stem cells might have greater more metastatic potential, since cells shed from the primary tumour would be more likely to be stem cells.

These considerations indicate that tumour size is a rather rough-and-ready indicator and that more refined molecular characterization of the cancer is likely to provide much more precise prognostic information.

We therefore propose that adding prognostically important suffixes, such as EIC (extensive intraductal component), PVI (perivascular invasion), M (multifocal), and inf (inflammatory) to the new $\mathrm{T}$ specification, which provide new information without compromising comprehensibility to either patient or clinician (e.g. pT1.5 EIC; pT0.7 $7_{\mathrm{PVI}}$ ).

We believe that a TNM workable classification should also be flexible enough to accommodate new information about prognostic indicators for breast cancer, including gene expression profiles. It is now possible to analyse the expression of thousands of genes simultaneously, using microarray techniques, and this has resulted in the identification of various sub-types of breast cancers, based on gene expression profile, that also show distinct clinical and prognostic features.

The two main breast cancer groups are defined by the presence or absence of ER. ER+ cancers are divided into luminal A and luminal B types according to gene expression profile, while ER - cancers are divided into normal breast-like, HER2+ and "basal-like".

The luminal B type has poorer prognosis than the luminal A type, but better prognosis than ER- cancers. ER - cancers have gene expression profiles (particularly basal epithelial-like cancers) similar to those of the basal epithelial cells or myoepithelial cells of healthy breast tissue, which express neither hormone receptors nor HER2 and have poor prognoses.

\section{Conflict of interest None.}

\section{References}

1. Denoix PF, Schwartz D (1959) General rules for classification of cancers and presentation of the therapeutic results. Mem Acad Chir (Paris) 85(15-16):415-424
2. Odicino F, Pecorelli S, Zigliani L, Creasman WT (2008) History of the FIGO cancer staging system. Int $\mathbf{J}$ Gynaecol Obstet 101(2):205-210

3. Greene FL, Page DL, Fleming ID, Fritz A, Balch CM, Haller DG, Morrow M (eds) (2002) AJCC cancer staging manual, 6th ed. Springer, New York, NY

4. Derogatis LR, Morrow GR, Fetting J, Penman D, Piasetsky S, Schmale AM, Henrichs M, Carnicke CL (1983) The prevalence of psychiatric disorders among cancer patients. JAMA 249(6):751-757

5. Leydon GM, Boulton M, Moynihan C, Alison J, Mossman J, Markella B, McPherson K (2000) Cancer Patients' information needs and information seeking behaviour: in depth interview study. Brit Med J 320(7239):909-913

6. Costelloe M, Nelson L (2004) The needs of recently diagnosed cancer patients. Nurs Stand 19(13):42-44

7. Veronesi U, Viale G, Rotmensz Goldhirsch A (2006) Rethinking TNM: breast cancer TNM classification for treatment decisionmaking and research. Breast 15(1):3-8

8. Veronesi U, Zurrida S, Viale G, Galimberti V, Arnone P, Nolè F (2009) Rethinking TNM: a breast cancer classification to guide to treatment and facilitate research. Breast J 3:291-295

9. Veronesi U, Zurrida S, Goldhirsch A, Rotmensz N, Viale G (2009) Breast cancer classification: time for a change. J Clin Oncol 27(15):2427-2428

10. Tavassoli FA (1998) Ductal carcinoma in situ: introduction of the concept of ductal intraepithelial neoplasia. Mod Pathol 11:140-154

11. Veronesi U, Paganelli G, Viale G, Luini A, Zurrida S, Galimberti V, Intra M, Veronesi P, Robertson C, Maisonneuve P, Renne G, De Cicco C, De Lucia F, Gennari R (2003) A randomized comparison of sentinel-node biopsy with routine axillary dissection in breast cancer. N Engl J Med 349:546-553

12. Veronesi U, Zurrida S, Mazzarol G, Viale G (2001) Extensive frozen section examination of axillary sentinel nodes to determine selective axillary dissection. World J Surg 25(6):806-808

13. Galimberti V (2006) International breast cancer study group trial of sentinel node biopsy. J Clin Oncol 24(1):210-211

14. Fernando HC (2008) Radiofrequency ablation to treat non-small cell lung cancer and pulmonary metastases. Ann Thorac Surg 85:S780-S784

15. Ranjan T, Abrey LE (2009) Current management of metastatic brain disease. Neurotherapeutics 6(3):598-603

16. Kennedy JE (2005) High-intensity focused ultrasound in the treatment of solid tumours. Nat Rev Cancer 5(4):321-327

17. Chiappa A, Makuuchi M, Lygidakis NJ, Zbar AP, Chong G, Bertani E, Sitzler PJ, Biffi R, Pace U, Bianchi PP, Contino G, Misitano P, Orsi F, Travaini L, Trifirò G, Zampino MG, Fazio N, Goldhirsch A, Andreoni B (2009) The management of colorectal liver metastases: expanding the role of hepatic resection in the age of multimodal therapy. Crit Rev Oncol Hematol 72(1):65-75

18. Paganelli G, Chinol M (2003) Radioimmunotherapy: is avidinbiotin pretargeting the preferred choice among pretargeting methods? Eur J Nucl Med Mol Imaging 30(5):773-776

19. Silvestrini R, Daidone MG, Mastore M, Di Fronzo G, Coradini D, Boracchi P, Squicciarini P, Salvadori B, Veronesi U (1993) Cell kinetics as a predictive factor in node-positive breast cancer treated with adjuvant hormone therapy. J Clin Oncol 6:1150-1155

20. Goldhirsch A, Wood WC, Gelber RD, Coates AS, Thürlimann B, Senn HJ (2007) Progress and promise: highlights of the international expert consensus on the primary therapy of early breast cancer. Ann Oncol 18(7):1133-1144 10th St. Gallen conference

21. Curigliano G, Viale G, Bagnardi PV, Fumagalli L, Locatelli M, Rotmensz N, Ghisini R, Zurrida S, Veronesi P, Goldhirsch A (2009) Clinical Relevance of HER2 Overexpression/amplification in patients with small tumor size and node-negative breast cancer. J Clin Oncol 27:5693-5699 
22. Jacobs TW, Gown AM, Yaziji H, Barnes MJ, Schnitt SJ (1999) Specificity of HercepTest in determining HER-2/neu status of breast cancers using the United States Food and Drug Administration-approved scoring system. J Clin Oncol 17:1983-1987

23. Persons DL, Tubbs RR, Cooley LD, Dewald GW, Dowling PK, Du E, Mascarello JT, Rao KW, Wilson KS, Wolff DJ, HabeggerVance G (2006) HER-2 fluorescence in situ hybridization: Results from the survey program of the College of American Pathologists. Arch Pathol Lab Med 130:325-331

24. Dal Lago L, Durbecq V, Desmedt C, Salgado R, Verjat T, Lespagnard L, Ma Y, Veys I, Di Leo A, Sotiriou C, Piccart M, Larsimont D (2006) Correction for chromosome 17 is critical for determination of true Her-2/neu gene amplification status in breast cancer. Mol Cancer Ther 5:2572-2579

25. Perou CM, Sørlie T, Eisen MB, van de Rijn M, Jeffrey SS, Rees CA, Pollack JR, Ross DT, Johnsen H, Akslen LA, Fluge OR, Pergamenschikov A, Williams C, Zhu SX, Lønning PE, Børresen-Dale AL, Brown PO, Botstein D (2000) Molecular portraits of human breast tumours. Nature 406:747-752

26. Sørlie T, Perou CM, Tibshirani R, Aas T, Geisler S, Johnsen H, Hastie T, Eisen MB, van de Rijn M, Jeffrey SS, Thorsen T, Quist $\mathrm{H}$, Matese JC, Brown PO, Botstein D, Eystein Lønning P, Børresen-Dale AL (2001) Gene expression patterns of breast carcinomas distinguish tumour subclasses with clinical implications. Proc Natl Acad Sci USA 98:10869-10874 